\title{
Melting and refreezing of zirconium observed using ultrafast x-ray diffraction
}

\author{
Harry B. Radousky $\odot,{ }^{1}, *$ Michael R. Armstrong, ${ }^{1}$ Ryan A. Austin, ${ }^{1}$ Elissaios Stavrou $\odot,{ }^{1}$ Shaughnessy Brown, ${ }^{2}$ \\ Alexander A. Chernov, ${ }^{1}$ Arianna E. Gleason, ${ }^{2,3}$ Eduardo Granados, ${ }^{2}$ Paulius Grivickas, ${ }^{1}$ Nicholas Holtgrewe, ${ }^{4}$ \\ Hae Ja Lee, ${ }^{2}$ Sergey S. Lobanov, ${ }^{4}$ Bob Nagler, ${ }^{2}$ Inhyuk Nam,${ }^{2}$ Vitali Prakapenka $\odot,{ }^{5}$ Clemens Prescher, ${ }^{6}$ Peter Walter, ${ }^{2}$ \\ Alexander F. Goncharov ${ }^{\oplus},{ }^{4}$ and Jonathan L. Belof ${ }^{1}$ \\ ${ }^{1}$ Lawrence Livermore National Laboratory, Physical and Life Sciences Directorate, \\ P.O. Box 808 L-350, Livermore, California 94550, USA \\ ${ }^{2}$ SLAC National Accelerator Laboratory, Menlo Park, California 94025, USA \\ ${ }^{3}$ Los Alamos National Laboratory, Los Alamos, New Mexico 87545, USA \\ ${ }^{4}$ Geophysical Laboratory, Carnegie Institution of Washington, Washington DC 20015, USA \\ ${ }^{5}$ Argonne National Laboratory, Argonne, Illinois 60439, USA \\ ${ }^{6}$ Institute for Geology and Mineralogy, University of Cologne, Cologne 50923, Germany
}

(Received 15 August 2019; revised manuscript received 23 December 2019; accepted 8 January 2020; published 24 February 2020)

\begin{abstract}
Ultrafast (130-fs) x-ray diffraction at the Linac Coherent Light Source has been applied to observe shock melting, which is driven by a rapid (120-ps) laser pulse impinging on a thin (few micrometers) bilayer of aluminum/zirconium. At a pressure of $100 \mathrm{GPa}$ in the aluminum (130 GPa in the zirconium), there is rapid melting of both metals and the recrystallization of zirconium into the bcc $\beta$ phase. We observe the solidification of the melt starting a few hundred picoseconds following the shock melting, out to $50 \mathrm{~ns}$ when the zirconium is fully crystallized into the bcc $\beta$ phase at a residual temperature of approximately $2000 \mathrm{~K}$. The pressure is obtained directly from the early time x-ray data, whereas the additional information from the x-ray line width and intensity at longer times inform a model of crystal nucleation and growth.
\end{abstract}

DOI: 10.1103/PhysRevResearch.2.013192

\section{INTRODUCTION}

Melting and freezing are one of the most common experiences [1], yet can be difficult to understand since the fundamental mechanisms occur on near-atomic and picosecond timescales [2-7]. Detailed knowledge of these mechanisms (and the ability to control them) has the potential to revolutionize how we view melting and freezing as well as applications, such as material synthesis of metallic glasses [6,7]. Since equilibration of metals upon rapid compression occurs on the timescale of the lattice vibrations, traditional long timescale (1-10-ns) compression experiments [8] cannot observe the melting transition mechanisms which are fundamentally a picosecond phenomena. More recently, several groups have looked at the melting of $\mathrm{Al}$ [9], Bi [10], and Sc [11] using a $>10$-ns laser. In particular, using a shock release method, Gorman et al. [10] observe melting on the timescale of $3 \mathrm{~ns}$. In order to study the fundamental aspects of melting, it is necessary to use laser pulses on the picosecond timescale. These measurements are accompanied by the need to have thinner samples. In particular, for shock speeds on

\footnotetext{
*radousky1@1lnl.gov

Published by the American Physical Society under the terms of the Creative Commons Attribution 4.0 International license. Further distribution of this work must maintain attribution to the author(s) and the published article's title, journal citation, and DOI.
}

the order of $5-10 \mathrm{~nm} / \mathrm{ps}$, a 100-ps duration laser drive will require a sample on the order of a few micrometers. These experiments also required the development of capabilities to analyze nonsteady shocks within the samples. In addition, velocimetry [12], which is conventionally used to estimate pressure and density of dynamically compressed samples in longer duration experiments, is not typically available at the picosecond timescale, which required the development of an ability to derive the shock pressure using the 100-fs x-ray data alone. Here, we report on results using the SLAC/Linac Coherent Light Source (LCLS) x-ray free-electron laser where zirconium was dynamically compressed to a pressure of $130 \mathrm{GPa}$, using an $\sim 120$-ps duration laser drive (20-ps rise time) and interrogated by 130 -fs duration $\mathrm{x}$-ray pulses.

Dynamic compression experiments [8], typically require timescales of several nanoseconds to ensure equilibration. Our fast compression experiments conducted at the LCLS Matter in Extreme Conditions (MEC) employed uniaxial compression, which accesses far-from-equilibrium mechanical states under extreme shear stress. Conventional picosecond timescale compression experiments can, by characterizing the flow of material, determine the time-dependent bulk stress/strain state within a sample. Such experiments have been used to observe a wide range of anomalous behavior, including extreme elastic deformation, simultaneous phase transformation and plasticity, and strain-rate-dependent shock-induced chemistry [13-16]. These previous experiments did not, however, have access to femtosecond x-ray diffraction and, therefore, could not provide a direct measure 


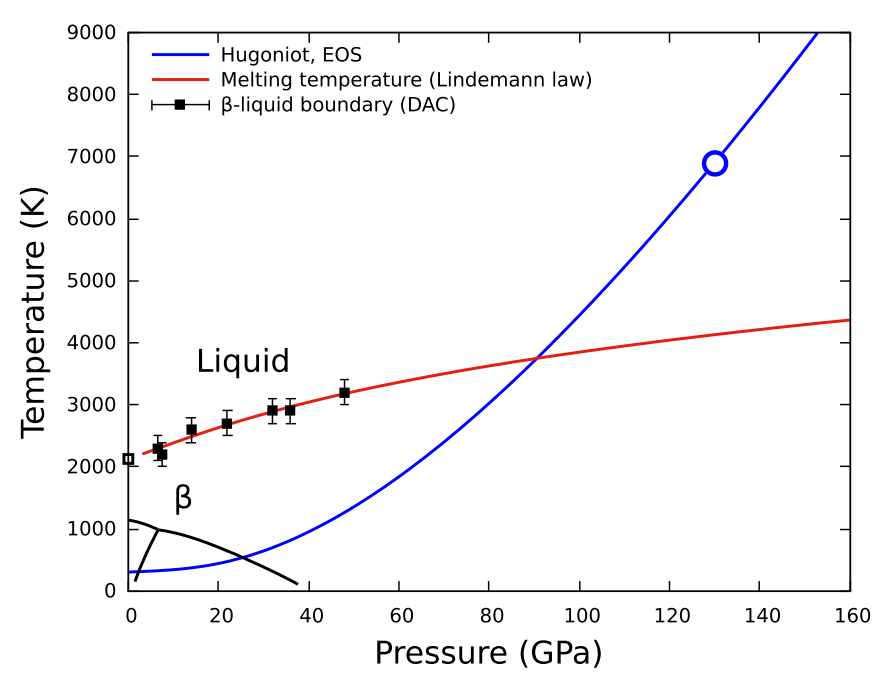

FIG. 1. Phase diagram for Zr. Solid-solid phase lines are taken from Ref. [17]. Melt line data, calculated melt curve, and the shock Hugoniot were determined as described in the text. The blue circle indicates the location of our data point.

of the atomic structure. $\mathrm{Zr}$ is well suited for studying material transformations due to its complex phase diagram allowing the study of three solid-solid phase transformations [17] plus the melt transition at pressures readily attainable at the MEC instrument [9]. Our studies of compression and release at the 100-ps timescale and 130-GPa pressure reveal melting within 100 ps of shock arrival, in-line with a recent study on Bi melting [18], and refreeze starting within hundreds of picoseconds after release. In addition, we follow the refreeze to $50 \mathrm{~ns}$ and observe substantial refreeze into the $\beta$ - $\mathrm{Zr}$ phase.

\section{EXPERIMENTAL AND COMPUTATIONAL METHODS}

The melting line of $\mathrm{Zr}$, under static high-pressure conditions, was determined in this paper using a laser-heated diamond-anvil cell) with micron-spatial resolution and a wellestablished time-resolved approach [19,20]. Details about the loading procedure, pressure determination, and temperature measurement can be found elsewhere [21,22]. The experimental results are shown in Fig. 1 together with a Lindemannderived melt line calculated in this paper, the $\mathrm{Zr}$ phase diagram and the $\mathrm{Zr}$ shock Hugoniot (a curve which gives thermodynamic variables for a range of final states subsequent to shock compression). Good agreement is observed between the experimentally determined and the Lindemann-derived melting lines. A recent study of the melting line of $\mathrm{Zr}$ under static conditions [23], using, essentially, the same method we used here gave a near-perfect agreement in the 30-50 GPa overlap range.

The $\mathrm{Zr}$ Hugoniot curve was calculated using an equation of state [24], which has been fit to shock compression data up to $140 \mathrm{GPa}$ [25]. This equation of state (EOS) does not distinguish among the different phases of $\mathrm{Zr}$ that may be present along the shock compression pathway. As such, the Hugoniot temperature is overestimated because the latent heats of transformation are neglected. From previous work [17], the latent heat of the $\alpha-\omega$ transformation is rather small, whereas the
Hugoniot temperature would be reduced by $\sim 500 \mathrm{~K}$ across the $\omega-\beta$ boundary. As discussed later, the latent heat of melting would reduce the temperature by another $\sim 400 \mathrm{~K}$. Therefore, the Hugoniot temperature predicted at $130 \mathrm{GPa}$ is too high by $\sim 900 \mathrm{~K}$ but still well above the extrapolated melt line.

An Al ablation layer and $\mathrm{Zr}$ sample layer were sputter coated on a SiN-coated Si wafer which had been chemically etched to give a $15 \times 15$ array of $550-\mu \mathrm{m}^{2} \mathrm{SiN}$ windows, spaced by $1 \mathrm{~mm}$. X-ray photoelectron spectroscopy (XPS) with $\mathrm{Al} K \alpha$ x rays was used for elemental compositional analysis. Each XPS measurement was obtained by an x-ray beam focused to a $200-\mu \mathrm{m}$ spot on a probed sample surface while gradually sputtering the surface with a $3-\mathrm{kV} \mathrm{Ar}{ }^{+}$ion beam. The absence of oxygen and Hf peaks in the full XPS spectra suggests that the $\mathrm{Zr}$ film was pure up to $0.1 \%$ in atomic mass (resolution of the XPS system). The 2.2- $\mu \mathrm{m} \mathrm{Al}$ ablator (coated on a thin $\mathrm{SiN}$ window) is driven by a $250-\mathrm{mJ} \sim 120$-ps FWHM duration laser pulse with a 20-ps initial rise, greater than a $30-\mu \mathrm{m}$ spot size launching a shock wave of similar duration into the ablator. The drive laser rise time is consistent with previous work [13-16]. The intensity contrast of the pulse is sufficient to avoid significant preheating $>100 \mathrm{~K}$ from estimates of the Al Bragg peaks shifts based on the known thermal EOS of $\mathrm{Al}$ at ambient pressure. We do not observe any thermal shift of the Al Bragg peaks between the static x-ray diffraction (XRD) patterns, acquired without the driving laser, and the XRD patterns acquired at early times (e.g., $-30 \mathrm{ps}$ ) before the laser drive. The shock wave transits the $\mathrm{Al}$ ablator and enters the $1.7-\mu \mathrm{m}$-thick $\mathrm{Zr}$ sample where rapid compression initiates a phase transition to a melted state in both the $\mathrm{Al}$ and in the Zr. After a variable delay, an $\sim 100$-fs duration $\mathrm{x}$-ray pulse with less than a 5- $\mu \mathrm{m}$ diameter cocentered with the drive laser is used to obtain an XRD pattern from the sample, providing structural information during the compression wave transit through the sample. Using this method on a set of identical samples by varying the delay between the laser drive and the $\mathrm{x}$-ray pulse, the progress of phase transformations in the $\mathrm{Zr}$ sample can be tracked with better than picosecond time resolution. This resulted in peak pressures of $100 \mathrm{GPa}$ in the $\mathrm{Al}$, corresponding to $130 \mathrm{GPa}$ in the $\mathrm{Zr}$, which is obtained by shock impedance matching with the Al ablator.

\section{RESULTS AND DISCUSSION}

Figure 2 shows the 2D x-ray images taken at $-30,600 \mathrm{ps,}$ and at $50 \mathrm{~ns}$, and the melting of both metals can be seen in the figure. At the initial $-30 \mathrm{ps}$, one can see both the ambient pressure rings for $\mathrm{Al}$ plus the rings for the hexagonal Zr. At 600 ps, both metals are melted with broad diffraction seen characteristic of melted Zr. At $50 \mathrm{~ns}$, the lines indicate recrystallization of the $\mathrm{Zr}$ into the body centered cubic (bcc) structure. The relatively broad Debye rings of $\mathrm{Zr}$ could be attributed to the the presence of residual stress/texturing due to the preparation method (sputtering) of the targets.

Selected one-dimensional integrated diffraction patterns are shown in Fig. 3. Subsequent to compression of $\mathrm{Zr}$, solid $\mathrm{Al}$ peaks are seen to be reduced and then disappear, indicating that $\mathrm{Al}$ had melted under shock compression [26]. Based on 

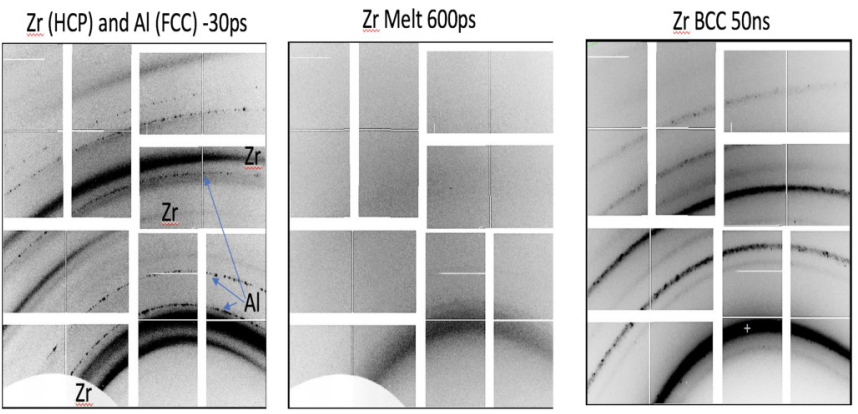

FIG. 2. X-ray diffraction two-dimensional (2D) images for $t=$ $-30,600 \mathrm{ps}$, and $50 \mathrm{~ns}$. At $600 \mathrm{ps}$, one observes diffraction features associated with melting of both $\mathrm{Zr}$ and $\mathrm{Al}$, followed by the recrystallization of $\mathrm{Zr}$ into the bcc structure.

linear variation of the ambient $\mathrm{Al}$ peak intensity with time (see Fig. 4), the shock wave speed in the Al ablator for 250-mJ drive energy was estimated to be $10.2 \mathrm{~km} / \mathrm{s}$ with an error of $10 \%$, corresponding to a shock pressure (assuming the known Hugoniot of Al) of $100 \mathrm{GPa}$.

To help determine the timing of compression and release waves in the sample and the distributions of pressure, planewave propagation in the $\mathrm{Al} / \mathrm{Zr}$ layers was simulated using a LLNL hydrodynamic simulation code. The compression wave was generated by a pressure boundary condition prescribed on the back surface of the Al layer with a peak amplitude and time variation selected to emulate the laser drive. The
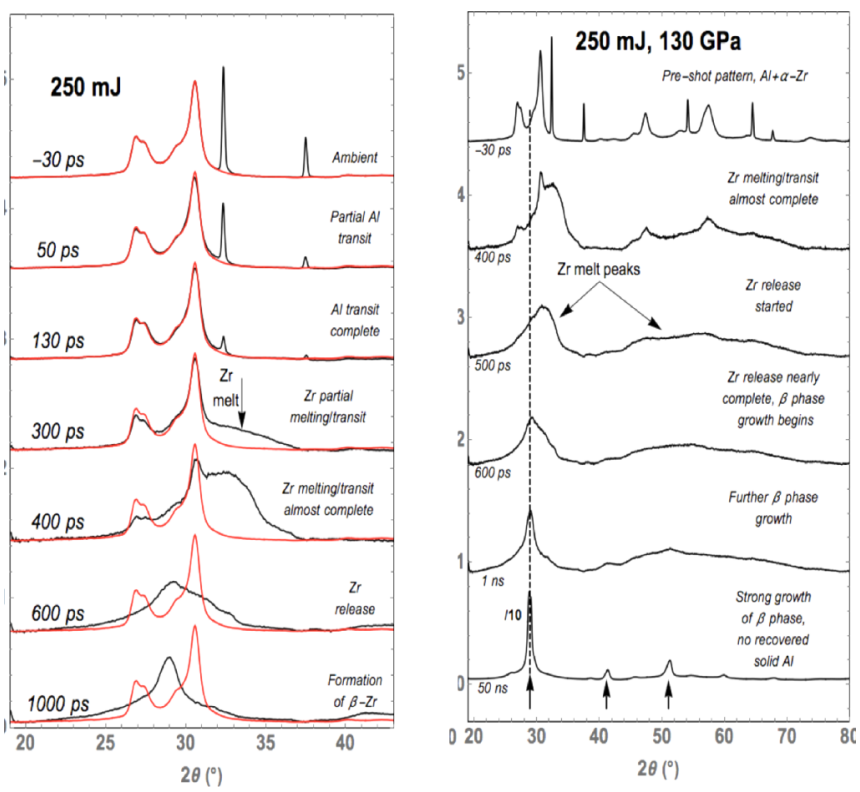

FIG. 3. X-ray diffraction as a function of increasing delay time. Each pattern represents a separate experiment on a set of identically prepared samples. The delay time varies in the left panel from before the pulse arrives $(-30 \mathrm{ps})$ to $1000 \mathrm{ps}$. In the right panel, delays vary from $400 \mathrm{ps}$ out to $50 \mathrm{~ns}$. The zirconium is under pressure and shock melted from approximately $300-600$ ps. From 600 ps out to $50 \mathrm{~ns}$, recrystallization occurs. The red traces are the $\mathrm{Zr}$ background with Al diffraction peaks removed for comparison to time-resolved data in black. The x-ray wavelength is $\lambda=1.301 \AA$. The Bragg peaks of the $\beta$-Zr phase are noted with arrows.
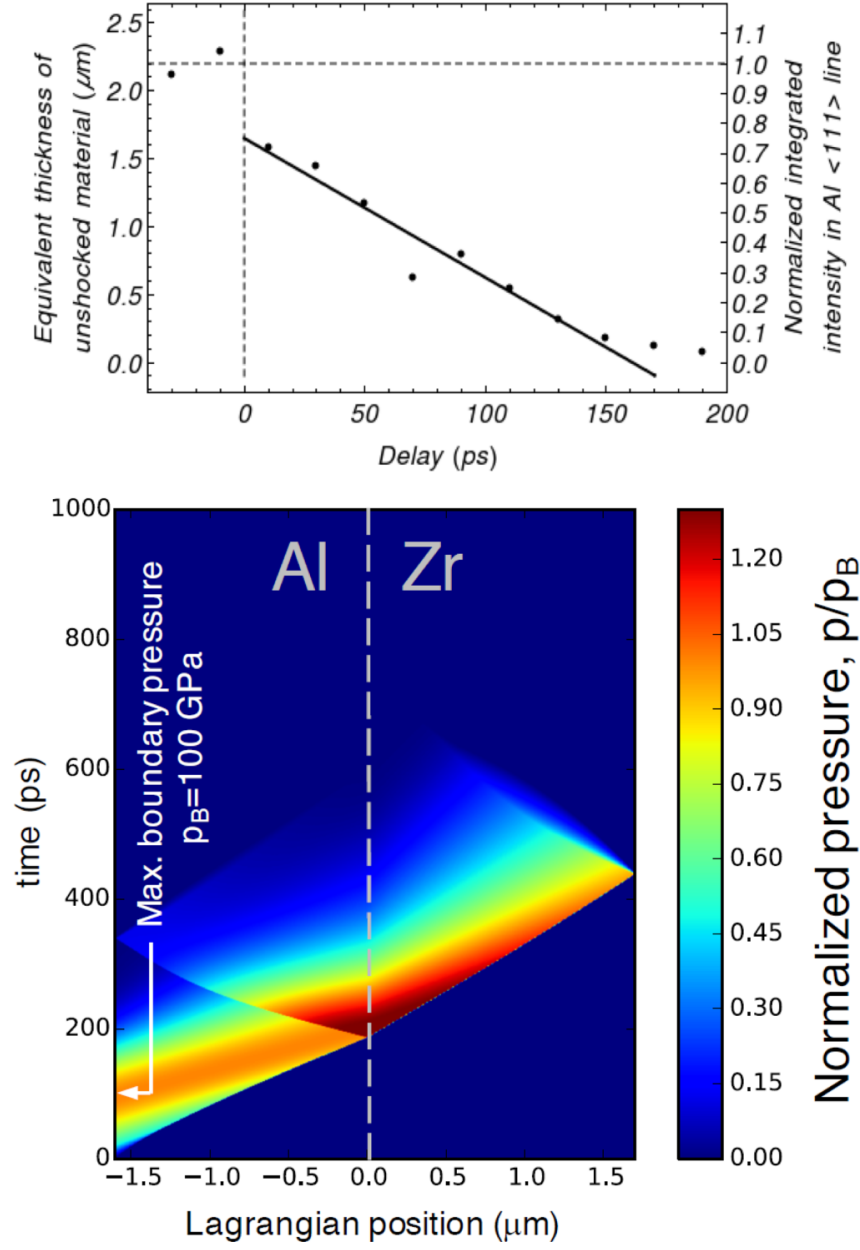

FIG. 4. The top panel shows the thickness of the unshocked Al ahead of the shock front, determined from the integrated area under the $\mathrm{Al}$ peaks. This gives the shock speed as $10.2 \mathrm{~km} / \mathrm{s}$. The bottom panel shows the simulated pressure field plotted on the space-time $(X-t)$ plane, which was obtained using a hydrocode calculation.

simulated thickness of the Al layer was reduced to the experimentally observed value at zero delay time $(1.6 \mu \mathrm{m})$ to account for ablation of material at the drive surface. The thermomechanical response of each layer $(\mathrm{Al}$ and $\mathrm{Zr}$ ) is described using a Mie-Gruneisen EOS and a constant heat capacity. Deviatoric stresses are neglected, and tensile pressures are not supported. The simulations employ the adiabatic assumption with temperatures computed from the difference between the internal energy and the cold energy. The selected EOS parametrizations reproduce the shock Hugoniot data for $\mathrm{Al}$ and $\mathrm{Zr}$ [25]. Here, we note that the equations of state do not distinguish among the different solid phases of $\mathrm{Zr}$, nor do they account for melting of either Al or Zr. In Fig. 4(b), the simulated pressure fields are plotted in the space of Lagrangian position $(X)$ versus time $(t)$. The pressure wave in $\mathrm{Al}$ steepens up before reflecting from the interface at 180 ps. A pressure wave with a peak amplitude of $130 \mathrm{GPa}$ then travels into the $\mathrm{Zr}$ layer. Release waves return the pressure in the $\mathrm{Zr}$ layer back to zero around 600-700 ps. Hydrodynamics simulations designed to emulate the experimental conditions, also shown in Fig. 4, illustrate the progress of the compression 


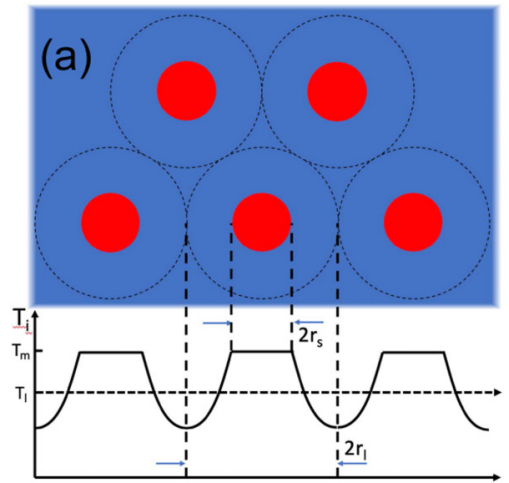

(b)

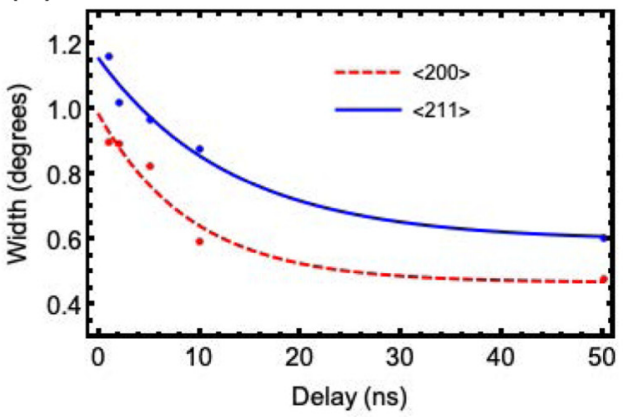

(c)

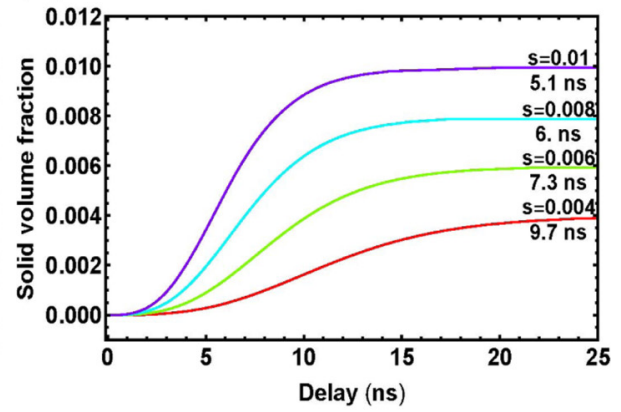

FIG. 5. (a) The pictorial model for crystal growth, with latent heat diffusing radially outward from the center of the solid nucleus and reducing the subsequent growth of neighboring crystallites. (b) The time-dependent variation of the experimental x-ray linewidths, which give a value for $r_{S}$ through the Scherrer equation, obtained by fitting a Lorentzian to the measured lineshapes. Panel (c) shows the integrated solid volume fraction from the heat-coupled, time-dependent crystal growth model, Eq. (3), as a function of time, at different values of initial dimensionless supercooling $s=\left(T_{m}-T_{0}\right) / T_{Q}$.

wave through the $\mathrm{Al} / \mathrm{Zr}$ sample as a function of time and match the observed times within the error bars. The rapid drop near $t=0$ of the Al lines is due to laser ablation, which launches the shock wave. This results in the loss of crystalline structure of $\mathrm{Al}$, see Fig. 3, over an approximately 500-nm thickness of material on the laser side of the $\mathrm{Al}$ ablator. The observed transit time accounts for transit through an ambient material, which is $500 \mathrm{~nm}$ thinner than the initial Al ablator thickness. This loss of crystallinity also provides information about ballistic electron thermal transport at very high fluence.

The remaining broad diffraction is consistent with liquid $\mathrm{Zr}$. We note that the melted $\mathrm{Al}$ at $400 \mathrm{ps}$ is released in pressure and would overlap the $\mathrm{Zr}$ melt peak which is still under pressure. It is expected that the majority of this broad peak derives from the $\mathrm{Zr}$ due to its higher scattering factor. In addition, no large $\mathrm{Al}$ melt peak is observed at later times once the $\mathrm{Zr}$ peak has shifted back to the lower angle. We also note that, at late times up to $50 \mathrm{~ns}$, the $\mathrm{Al}$ peaks do not reappear. This implies that $\mathrm{Al}$ remains melted over this time period, which is consistent with its low melting temperature compared to $\mathrm{Zr}$, and the high residual temperature of the $\mathrm{Zr}$ out to late times due to the latent heat of recrystallization.

Shock melting of $\mathrm{Zr}$ is evident within $\sim 100$ ps ( 300-ps absolute time) after shock arrival in $\mathrm{Zr}$ via the appearance of broad pressure-shifted peaks consistent with a compressed liquid. Ambient $\mathrm{Zr}$ peaks decrease in intensity at subsequent delays as the shock transits the $\mathrm{Zr}$ sample. Partial release at 500 ps shifts the broad peaks to lower angles followed by nearly complete release at 600 ps and evidence of initial growth of $\beta-\mathrm{Zr}$. Further growth of $\beta-\mathrm{Zr}$ occurs on nanosecond timescales where much stronger diffraction peaks at $50 \mathrm{~ns}$ likely indicate high crystallinity growth from the liquid phase.

Our sample contains less than $0.1 \%$ (atomic) of both oxygen and hafnium meaning the melt line will not be significantly shifted. We clearly observe the melting of $\mathrm{Zr}$ at the shock pressure corresponding to $130 \mathrm{GPa}$ in the $\mathrm{Zr}$. Melting at this shock pressure is consistent with crossing the melting line shown in Fig. 1. Of note is the recrystallization of the $\beta$ phase, existing to late times of, at least, $50 \mathrm{~ns}$. The data show a very strong diffraction signature compared to the original $\alpha-\mathrm{Zr}$ phase, which indicates a new texturing as it grows from the melt phase. From both the higher angle-shifted location of the diffraction peak and the timing, $\mathrm{Zr}$ is melting under compression. Also, at 50 and $130 \mathrm{ps,} \mathrm{we} \mathrm{observe} \mathrm{a}$ substantial decrease in the intensity of the ambient $\mathrm{Al}\langle 111\rangle$ peak, suggesting (along with the estimated 1-Mbar shock pressure) that the $\mathrm{Al}$ is shock melted on compression and stays melted upon reflection of the shock at the $\mathrm{Al} / \mathrm{Zr}$ interface. The presence of broad melt peaks in both $\mathrm{Al}$ and $\mathrm{Zr}$ is consistent with the estimate of shock pressure in the $\mathrm{Al}$ ablator given by the shock speed from Fig. 4.

As shown in Fig. 3, the $\mathrm{Zr}$ melting occurs between the times of about 300 and 600 ps, thus, lasting 300 ps. Crystallization begins $\sim 200$ ps after melting and crystallization was followed out to a very long time of $50 \mathrm{~ns}$. Our simulation of the shock wave propagation through the sample, Fig. 4 (the right panel), shows that the pressure in the sample releases to zero pressure at about 600-700 ps. This is compared to the time for crystallization which is observed out to $50 \mathrm{~ns}$. This quick cooling followed by a long crystallization time is analogous to the temperature time plateau observed by calorimetry in conventional crystallization at ambient conditions. This plateau comes from the latent heat and is self-consistently maintained since the external cooling is compensated by the heat generated during crystallization in the melt-crystal slurry.

The average crystallite size at time $t$ follows from the $\beta-\mathrm{Zr}$ crystal diffraction linewidths $w(t)$, whereas the ratio of the diffraction intensity at a given moment of time to the maximal intensity reached at $50 \mathrm{~ns}$ can be used as a measure of the solidified part in the sample by that time under the assumption that by $50 \mathrm{~ns}$ the solidification is substantially complete. The data are shown in Fig. 5.

The Scherrer equation provides the average radius of crystallites $r_{s}(t)$

$$
r_{s}(t)=\frac{K \lambda}{\left[w(t)-w_{\text {in }}\right] \cos \theta} .
$$

Here, $w$ is the width of the diffraction line at its half maximum in radians at the diffraction angle $\theta$ whereas $w_{\text {in }}\left(=0.1^{\circ}\right)$ is the instrumental linewidth. The radiation 
wavelength is $\lambda=1.3 \AA$. For the spherical crystallites, the constant $K=1.11$. Equation (1) for $t=1 \mathrm{~ns}$ results in crystal radii $r_{S}=7.6,5.5$, and $4.3 \times 10^{-7} \mathrm{~cm}$ for the lines diffracting at $\theta=14.4^{\circ}, 20.65^{\circ}$, and $25.5^{\circ}$, respectively. At $t \leqslant 10 \mathrm{ns,}$ the diffraction intensity shows that the solid fraction $\phi \ll 1$. Therefore, we assume that each crystal sphere sits in the center of a concentric liquid cell with radius $r_{L}=\phi^{-1 / 3} r_{s}$ remaining constant in time. The cells form a lattice [Fig. 5(a)] and are taken to be equal, making each thermally isolated. Comparing the crystal radii at $t=1$ and $2 \mathrm{~ns}$, one may estimate the average growth rate to be $V \approx 150 \mathrm{~cm} / \mathrm{s}$. At the later times, the growth rate decreases.

The growth rate of $150 \mathrm{~cm} / \mathrm{s}$ estimated above allows an estimate of the average supercooling in the slurry. Since the zirconium entropy of fusion is low [27-29], $\Delta S=1.04 k$ (where $k$ is the Boltzmann constant), the crystal-melt interface is assumed to be disordered. Therefore, the growth rate $V$ is given by Eq. (2) and is proportional to the crystallization driving force $\Delta \mu / k T_{i}$ where $\Delta \mu$ is the difference between the liquid and the solid chemical potentials. $T_{m}$ is the melting temperature at ambient pressure and $T_{i}$ is the temperature at the interface [30,31]

$$
V=\frac{\beta \Delta \mu}{k T_{i}} \equiv \beta_{T}\left(T_{m}-T_{i}\right) .
$$

In the numerical estimates below, we distinguish the interface temperature $T_{i}$ from the bulk melt temperature $T$ only when it enters the interface supercooling and is multiplied by the large kinetic coefficient $\beta=\left(\frac{k T_{i}}{m}\right)^{1 / 2}=4 \times 10^{4} \mathrm{~cm} / \mathrm{s}$ determining the growth rate [31] $\beta_{T}=19 \mathrm{~cm} / \mathrm{s} \mathrm{K}$.

Using the cell model, the cooling process may be separated into two parts. The first is adiabatic cooling due to the release of pressure in a thermally isolated cell. Its initial temperature may be $T_{m}$ if crystal seeds are present or is much lower, down to $0.7 T_{m}$ if homogeneous nucleation is possible $[32,33]$. The seeded or nucleated crystals grow until the supercooling falls to zero. This supercooling depends on the initial temperature, total adiabatic temperature drop, and on the latent heating temperature $T_{Q}=T_{m} \Delta S / c_{P}$, the ratio of the latent heat to the heat capacity. For $\mathrm{Zr}$, we take the temperature rise due to latent heat to be $T_{Q}=423 \mathrm{~K}$ based on previously measured values at ambient conditions [34]. This is the temperature rise in a melt of elemental $\mathrm{Zr}$ crystallized under thermally and mechanically isolated conditions. Unless the initial temperature is deep below the melting point, the final cell temperature reaches the melting point before the whole melt is crystallized. That low undercooling case is probably realized in these experiments and the second part of the cooling begins. This part is controlled by the latent heat transport out of the whole sample rather than the small cell. Therefore, that second part may last much longer.

A crystallite of a nanoscale radius $r_{S} \approx 10^{-6} \mathrm{~cm}$ or smaller growing in a supercooled melt is expected to be heated close to the melting point within the time of $\sim\left(r_{S}^{2}\right) / 4 \alpha \approx 2$ ps where the $\mathrm{Zr}$ thermal diffusivity $\alpha \approx 0.12(\mathrm{~cm})^{2} / \mathrm{s}$ [35]. Later, the growing crystal has nearly no ability to absorb more latent heat so that all latent heat goes to the melt. During the initial adiabatic part of the process, the average liquid temperature $\overline{T_{l}}$ is controlled by the simplified heat balance equation within the cell, coupled with the crystal growth rate $\frac{d r_{s}}{d t}$ and with a driving force supplied by the rate of undercooling, $g(t)$ :

$$
\begin{aligned}
\frac{d \overline{T_{l}}}{d t} & =\frac{3 T_{Q} r_{S}^{2}}{r_{l}^{3}-r_{S}^{3}} \frac{d r_{S}}{d t}-g(t), \\
\frac{d}{d t}\left(\frac{r_{S}}{r_{l}}\right) & =\frac{\beta_{T}}{r_{l}}\left(T_{m}-T_{i}\right) \approx \frac{\beta_{T}}{r_{l}}\left(T_{m}-\overline{T_{l}}\right) \\
& =\frac{1}{\tau}\left\{\frac{T_{m}-T_{0}}{T_{Q}}+\ln \left[1-\left(\frac{r_{S}}{r_{l}}\right)^{3}\right]\right\} .
\end{aligned}
$$

The second line of Eq. (3) follows from Eq. (2) where the crystal interface temperature $T_{i}(t)$ is approximated by the integral $\bar{T}_{l}(t)$ obtained from the first line of Eq. (3). The integration constant $T_{0}$ is the melt temperature at $t=0$ plus the integral of $g(t)$ over $t>0$, both unknown. The solution of the second line of Eq. (3) is presented in Fig. 5(c) for several initial constant values of the supercooling $s=\left(T_{m}-T_{0}\right) / T_{Q}$. The characteristic timescale for the process $\tau \equiv r_{L} / \beta_{T} T_{Q}=$ $300 \mathrm{ps}$ where the numerical estimate is for the experimental $r_{L} \cong 2.4 \times 10^{-6} \mathrm{~cm}$. Cessation of the fast growth at $6 \mathrm{~ns}$ suggests the transition to latent heat being transported over the whole sample rather than the small cell. Further sample cooling due to radiation may require microseconds to occur, which is beyond the timescale of relevance to this system.

We observed melting of metals on the timescale of a few hundred picoseconds, and we observed the recrystallization of $\mathrm{Zr}$ on timescales up to $50 \mathrm{~ns}$. The shock speed in $\mathrm{Al}$ was determined entirely from the x-ray diffraction patterns, allowing us to determine the pressure in $\mathrm{Zr}$ from impedance matching. The latent heat of crystallization is found to provide the energy for the recrystallization process to extend out to $50 \mathrm{~ns}$ following a 120 -ps pulse which drove the initial shock melting. The fact that we observe refreeze rather than a metallic glass indicates that the formation of single phase metallic glasses, even with these extremely high cooling rates, may be fundamentally limited.

\section{ACKNOWLEDGMENTS}

This work was performed under the auspices of the U.S. Department of Energy (DOE) by Lawrence Livermore National Security, LLC under Contract No. DE-AC5207NA27344. We gratefully acknowledge the LLNL LDRD Program for funding support of this Project under Project No. 16-ERD-037. A.F.G. and N.H. acknowledge support from the Army Research Office (Grants No. 56122-CH-H and No. 71650-CH) and the Deep Carbon Observatory. Portions of this work were performed at GeoSoilEnviroCARS (The University of Chicago, Sector 13), Advanced Photon Source (APS), Argonne National Laboratory. GeoSoilEnviroCARS is supported by the National Science Foundation Earth Sciences (Grant No. EAR1634415) and Department of Energy-GeoSciences (Grant No. DE-FG02-94ER14466). This research used resources of the Advanced Photon Source, a U.S. DOE Office of Science User Facility operated for the DOE Office of Science by Argonne National Laboratory under Contract No. DE-AC02-06CH11357. Use of the Linac Coherent Light Source (LCLS), SLAC National Accelerator Laboratory, is supported by the U.S. Department of Energy, 
Office of Science, Office of Basic Energy Sciences under Contract No. DE-AC02-76SF00515. The MEC instrument is supported by the U.S. Department of Energy, Office of Science, Office of Fusion Energy Sciences under Contract No.
SF00515. One of the authors (H.B.R.) thanks Dr. J. Berry for helpful discussions. A.E.G. acknowledges LANL LDRD Reines support. We thank Dr. J. Crowhurst for his help with the sample preparation.
[1] M. A. Sánchez, T. Kling, T. Ishiyama, M.-J. v. Zadel, P. J. Bisson, M. Mezger, M. N. Jochum, J. D. Cyran, W. J. Smit, H. J. Bakker, M. J. Shultz, A. Morita, D. Donadio, Y. Nagata, M. Bonn, and E. H. G. Backus, Proc. Natl. Acad. Sci. USA 114, 227 (2017).

[2] V. I. Levitas and R. Ravelo, Proc. Natl. Acad. Sci. USA 109, 13204 (2012).

[3] E. K. Cerreta, G. T. Gray, C. P. Trujillo, D. W. Brown, C. N. Tomé, M. Elert, M. D. Furnish, R. Chau, N. Holmes, and J. Nguyen, in Proceedings of the Conference of the American Physical Society Topical Group on Shock Compression of Condensed Matter, edited by M. Elert, M. D. Furnish, R. Chau, N. Hollmes, and J. Nguyen, AIP Conf. Proc. No. 955 (AIP, New York, 2007), p. 635.

[4] J. Zhang, Y. Zhao, C. Pantea, J. Qian, L. L. Daemen, P. A. Rigg, R. S. Hixson, C. W. Greeff, G. T. Gray III, Y. Yang, L. Wang, Y. Wang, and T. Uchida, J. Phys. Chem. Solids 66, 1213 (2005).

[5] M. T. Pérez-Prado and A. P. Zhilyaev, Phys. Rev. Lett. 102, 175504 (2009).

[6] Y. Ju, Q. Zhang, Z. Gong, G. Ji, and L. Zhou, J. Appl. Phys. 114, 093507 (2013).

[7] J. Zhang and Y. Zhao, Nature (London) 430, 332 (2004).

[8] W. Nellis, Ultracondensed Matter by Dynamic Compression (Cambridge University Press, Cambridge, UK, 2017).

[9] L. B. Fletcher, H. J. Lee, T. Döppner, E. Galtier, B. Nagler, P. Heimann, C. Fortmann, S. LePape, T. Ma, M. Millot, A. Pak, D. Turnbull, D. A. Chapman, D. O. Gericke, J. Vorberger, T. White, G. Gregori, M. Wei, B. Barbrel, R. W. Falcone, C.-C. Kao, H. Nuhn, J. Welch, U. Zastrau, P. Neumayer, J. B. Hastings, and S. H. Glenzer, Nat. Photon. 9, 274 (2015).

[10] M. G. Gorman, R. Briggs, E. E. McBride, A. Higginbotham, B. Arnold, J. H. Eggert, D. E. Fratanduono, E. Galtier, A. E. Lazicki, H. J. Lee, H. P. Liermann, B. Nagler, A. Rothkirch, R. F. Smith, D. C. Swift, G. W. Collins, J. S. Wark, and M. I. McMahon, Phys. Rev. Lett. 115, 095701 (2015).

[11] R. Briggs, M. G. Gorman, A. L. Coleman, R. S. McWilliams, E. E. McBride, D. McGonegle, J. S. Wark, L. Peacock, S. Rothman, S. G. Macleod, C. A. Bolme, A. E. Gleason, G. W. Collins, J. H. Eggert, D. E. Fratanduono, R. F. Smith, E. Galtier, E. Granados, H. J. Lee, B. Nagler, I. Nam, Z. Xing, and M. I. McMahon, Phys. Rev. Lett. 118, 025501 (2017).

[12] S. B. Brown, A. Hashim, A. Gleason, E. Galtier, I. Nam, Z. Xing, A. Fry, A. MacKinnon, B. Nagler, E. Granados, and H. J. Lee, Rev. Sci. Instrum. 88, 105113 (2017).

[13] J. C. Crowhurst, M. R. Armstrong, K. B. Knight, J. M. Zaug, and E. M. Behymer, Phys. Rev. Lett. 107, 144302 (2011).

[14] M. R. Armstrong, J. M. Zaug, N. Goldman, I.-F. W. Kuo, J. C. Crowhurst, W. M. Howard, J. A. Carter, M. Kashgarian,
J. M. Chesser, T. W. Barbee Jr., and S. Bastea, J. Phys. Chem. A 117, 13051 (2013).

[15] J. C. Crowhurst, B. W. Reed, M. R. Armstrong, H. B. Radousky, J. A. Carter, D. C. Swift, J. M. Zaug, R. W. Minich, N. E. Teslich, and M. Kumar, J. Appl. Phys. 115, 113506 (2014).

[16] M. R. Armstrong, P. V. Grivickas, A. M. Sawvel, J. P. Lewicki, J. C. Crowhurst, J. M. Zaug, H. B. Radousky, E. Stavrou, C. T. Alviso, J. Hamilton, and R. S. Maxwell, J. Polym. Sci. Part B: Polym. Phys. 56, 827 (2018).

[17] C. W. Greeff, Modell. Simul. Mater. Sci. Eng. 13, 1015 (2005).

[18] C. M. Pépin, A. Sollier, A. Marizy, F. Occelli, M. Sander, R. Torchio, and P. Loubeyre, Phys. Rev. B 100, 060101(R) (2019).

[19] S. Anzellini, A. Dewaele, M. Mezouar, P. Loubeyre, and G. Morard, Science 340, 464 (2013).

[20] A. Dewaele, M. Mezouar, N. Guignot, and P. Loubeyre, Phys. Rev. Lett. 104, 255701 (2010).

[21] E. Stavrou, L. H. Yang, P. Söderlind, D. Aberg, H. B. Radousky, M. R. Armstrong, J. L. Belof, M. Kunz, E. Greenberg, V. B. Prakapenka, and D. A. Young, Phys. Rev. B 98, 220101(R) (2018).

[22] V. B. Prakapenka, A. Kubo, A. Kuznetsov, A. Laskin, O. Shkurikhin, P. Dera, M. L. Rivers, and S. R. Sutton, High Pressure Res. 28, 225 (2008).

[23] P. Parisiades, F. Cova, and G. Garbarino, Phys. Rev. B 100, 054102 (2019).

[24] D. J. Steinberg, Equation of State and Strength Properties of Selected Materials, Lawrence Livermore National Laboratory Technical Report No. UCRL-MA-106439, 1996 (unpublished).

[25] S. P. Marsh, LASL Shock Hugoniot Data (University of California Press, Berkeley, CA, 1980).

[26] J. A. Moriarty, D. A. Young, and M. Ross, Phys. Rev. B 30, 578 (1984).

[27] V. N. Korobenko and A. I. Savvatimskii, High Temp. 39, 659 (2001).

[28] V. N. Korobenko and A. I. Savvatimskii, High Temp. 39, 525 (2001).

[29] V. N. Korobenko and A. I. Savvatimskii, High Temp. 39, 485 (2001).

[30] A. A. Chernov, Modern Crystallography III. Crystal Growth, Pt I. Springer Solid State Sci. Vol. 36 (Springer, Berlin, 1984).

[31] L. Mikheev and A. Chernov, J. Cryst. Growth 112, 591 (1991).

[32] P. C. Myint, A. A. Chernov, B. Sadigh, L. X. Benedict, B. M. Hall, S. Hamel, and J. L. Belof, Phys. Rev. Lett. 121, 155701 (2018).

[33] P. C. Myint and J. L. Belof, J. Phys.: Condens. Matter 30, 233002 (2018).

[34] J. J. Valencia and P. N. Quested, in ASM Handbook (ASM International, Cleveland, OH, 2008), Vol. 15, pp. 468-481.

[35] J. Fink and L. Leibowitz, J. Nucl. Mater. 226, 44 (1995). 